Provided for non-commercial research and education use. Not for reproduction, distribution or commercial use.

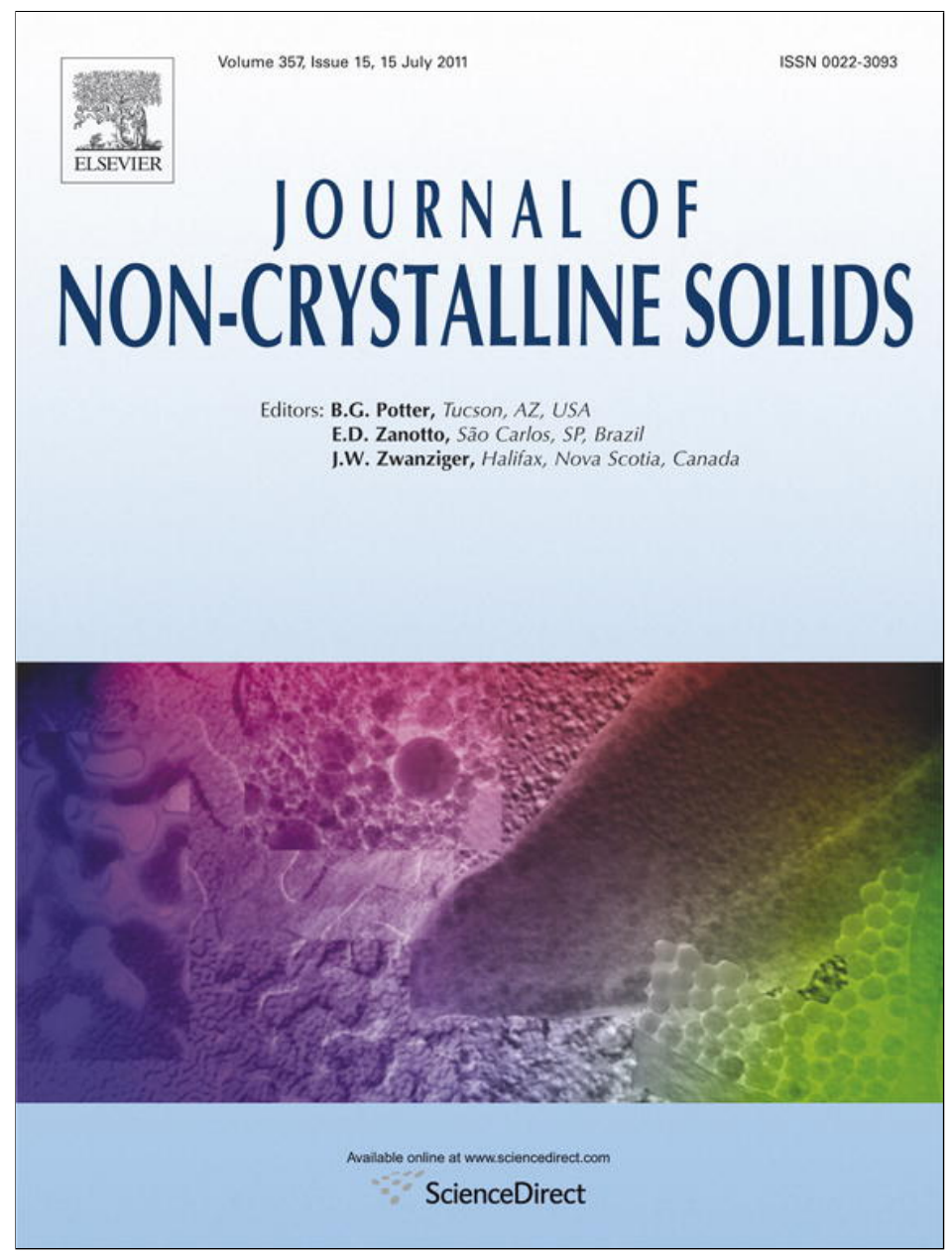

This article appeared in a journal published by Elsevier. The attached copy is furnished to the author for internal non-commercial research and education use, including for instruction at the authors institution and sharing with colleagues.

Other uses, including reproduction and distribution, or selling or licensing copies, or posting to personal, institutional or third party websites are prohibited.

In most cases authors are permitted to post their version of the article (e.g. in Word or Tex form) to their personal website or institutional repository. Authors requiring further information regarding Elsevier's archiving and manuscript policies are encouraged to visit:

http://www.elsevier.com/copyright 


\title{
Dissolution retardation of solid silica during glass-batch melting
}

\author{
Pavel Hrma*, José Marcial \\ Pacific Northwest National Laboratory, 99352 Richland, Washington, USA
}

\section{A R T I C L E I N F O}

\section{Article history:}

Received 9 February 2011

Received in revised form 30 March 2011

Available online 19 April 2011

\section{Keywords:}

Glass formation;

Quartz;

Melting.

Borosilicates;

Diffusion

\begin{abstract}
A B S T R A C T
During glass-batch melting, solid silica (quartz) usually dissolves last. The measured rate of dissolution, while the temperature was increasing at a constant rate, was compared with the hypothetical diffusion-controlled volume flux from regularly distributed particles through concentration layers of a uniform thickness. The actual rate was up to two orders of magnitude lower than that of the "ideal" case, revealing a progressive inhibition of silica dissolution. As a measure of this retarded dissolution, we introduced a retardation factor defined as a ratio of the actual and "ideal" dissolution rates measured as the volume flux at the melt-particle interface. The severe inhibition of silica dissolution has been attributed to the irregular spatial distribution of silica particles that is associated with the formation of nearly saturated melt on a portion of their surfaces. Irregular shapes and unequal sizes of particles also contribute to their extended lifetime.
\end{abstract}

(c) 2011 Elsevier B.V. All rights reserved.

\section{Introduction}

Reactions between quartz particles and the other glass-batch components have been studied by numerous researchers [1]. By definition, the batch-to-glass conversion process ends when the residual quartz particles (from quartz sand or crushed crystals) dissolve in the glass-forming melt via multicomponent diffusion of silica against the remaining glass components.

The first steps toward understanding the diffusion-controlled dissolution of quartz particles were to solve the pertinent field equations for a single spherical particle in a quiescent melt while ignoring the effects of gravity [2-5]. Using a boundary-layer approach, Levich [6] obtained an analytical solution for a particle moving by buoyancy. The dissolution of multiple particles was described for stirred liquids $[7,8]$ and later adapted to the case of sand grains in molten glass when the silica concentration in the melt was close to the solubility limit [9].

These developments assumed a constant temperature during the particle lifetime. In reality, the batch contains multiple particles randomly distributed in space that react and dissolve while the temperature is increasing. Moreover, the particle sizes are distributed within a certain range, the particle shapes are non-spherical, and their surfaces can be rough. Also, the melt is moving, driven by the combination of natural and forced convection and by the nucleation, growth, and motion of gas bubbles. The interaction of bubbles and particles is crucial for the glass-melting process. As Nemec et al. [1012] have shown, bubbles nucleate on dissolving solid particles, and their formation ceases as soon as the particles are fully dissolved.

* Corresponding author at: Currently at the Division of Advanced of Nuclear Engineering, POSTECH, Pohang, Republic of Korea.

E-mail address: pavel.hrma@pnl.gov (P. Hrma).
In a previous paper [1], experimental data indicated that the dissolution rate did not increase with increasing temperature as rapidly as one would expect. In fact, the dissolution was increasingly hindered as the conversion progressed. We have attributed this retardation to the mutual interactions of neighboring particles, namely, as Fig. 1 illustrates, to the impinging of concentration layers that grow around dissolving particles. We proposed that the diffusion layers grow, and the bulk concentration of silica remains constant initially. Then the bulk concentration increases, approaching saturation, while convection keeps the diffusion layers at a constant average thickness.

However, the final silica concentration of a fully homogenized melt is below the solubility limit. The melt can be fully saturated with silica only on a limited, though increasing, portion of melt volume as a result of nonuniform distribution of particles in space. Other contributing factors include the particle shapes, particle-size distribution, and the stirring of the mixture by bubbles.

To quantify the dissolution retardation, we have defined a dissolution retardation factor that is based on a notion of ideal batch in which equally sized spherical particles are uniformly distributed in space, and the stirring effect of bubbles keeps the concentration layers at a uniform thickness.

\section{Formulation}

\subsection{Ideal batch}

Models are constructed to represent salient features of objects or processes while disregarding details that may modify, but do not determine, the features of interest. Models are useful for understanding phenomena occurring in complex situations by formulating 


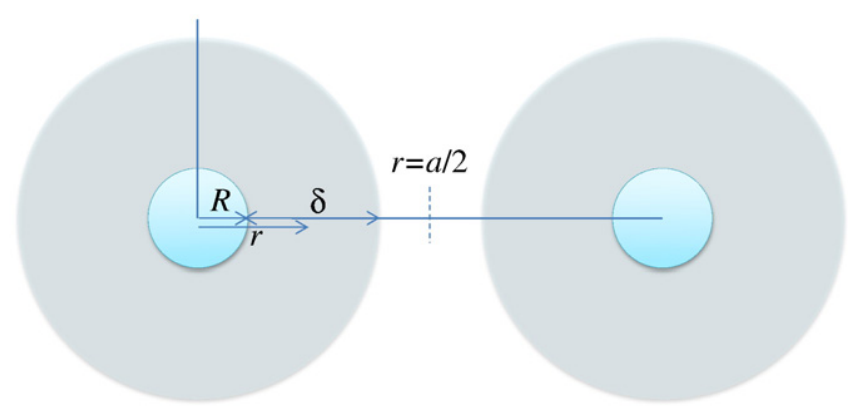

Fig. 1. Dissolving spherical particles of radius $R$ with concentration layers of thickness $\delta$. As the particles continue to dissolve, $R$ decreases and $\delta$ increases until the concentration layers impinge on each other. This impedes further dissolution. An example of the concentration distribution within the concentration layers is shown in Fig. 7.

constitutive relationships characteristic of the responses of the system to the controlling variables.

Glass-batch melting is an example of such a complex process. Here we construct an "ideal" batch containing solid silica of equally sized spherical particles that are uniformly distributed within the mixture. The particles dissolve uniformly via diffusion, and the concentration fields around them are sole functions of the radial distance from particle surfaces.

These features contrast with reality, where the particles are nonspherical, polydisperse (though within a narrow range of sizes), nonuniformly distributed in space, and surrounded by a highly nonuniform concentration field. The dissolution retardation factor, mentioned above and defined below, quantifies the difference between the ideal and a real batch, or - in other words - gages the degree of "non-ideality" of the real batch.

In the ideal batch, the dissolving particles of solid silica are spheres of equal radii, uniformly arranged on a cubic network. For simplicity, we assume that the particles and melt are of equal, uniform density and that the space is completely filled with the particles and melt. Although bubbles stir the melt, we omit the volume they occupy. Then the average distance $(a)$ between each of the six neighboring particles is

$a=\left(\frac{4 \pi}{3 c_{F}}\right)^{\frac{1}{3}} R_{0}$

where $R_{0}$ is the initial particle radius, and $\mathrm{c}_{\mathrm{F}}$ is the final mass fraction of silica in the homogeneous glass, i.e., after all particles dissolve and inhomogeneities disappear.

\subsection{Degree of stirring and bulk concentration}

Because the mixing is controlled by diffusion, the dissolution of solid particles is affected by convection. Although both forced and natural convection operate on a large scale (circulation flows in a glass melter, driven by gravity or forced by bubbling), it is mainly on a scale comparable with the size of concentration inhomogeneities in which natural convection affects the dissolving particles. Solid particles rise or sink, depending on their density and the degree and type of agglomeration while growing and rising bubbles move upward through a nonuniform concentration that induces convection driven by surface tension gradients. By effectively stirring the melt, bubbles help homogenization, even though they also can push the dissolving particles into agglomerates and drag the particles and agglomerates to the melt surface. These various effects impact the overall process in an observable, even measurable, manner.

The stirring effect of bubbles suppresses the concentration layers around the particles to various degrees. We consider here two special cases of the evolution of concentration layers. First, the concentration layers impinge on each other and then remain at a constant thickness
( $\delta)$. Second, the concentration layers of neighboring particles remain at the same distances while the particles are dissolving so that $R+3 \delta=$ constant, where $R$ is the particle radius. We associate the former case with vigorous stirring and the latter with mild stirring.

For vigorous stirring, we estimate the bulk mass fraction, $c_{\mathrm{B}}$, using the formula

$c_{B}=\frac{c_{F}-c_{S}}{1-c_{S}}$

where $c_{S}$ is the mass fraction of undissolved silica in the mixture of quartz particles and melt. Eq. (2a) follows from the mass balance of silica requiring that the sum of dissolved and undissolved silica is constant, i.e., $c_{\mathrm{B}}\left(1-c_{\mathrm{S}}\right)+c_{\mathrm{S}}=\mathrm{c}_{\mathrm{F}}$, and thus identifies the $c_{\mathrm{B}}$ with the average mass fraction of silica in the melt, ignoring the excess silica within the concentration layers. This is acceptable when stirring is vigorous, and concentration layers are thin.

With a mild stirring, a larger fraction of silica is retained in the concentration layers, and thus $c_{\mathrm{B}}$ is lower than the average defined by Eq. (2a). In this case, somewhat arbitrarily, we chose the Kolmogorov function for the $c_{\mathrm{B}}(t)$, where $t$ is the time

$c_{B}=c_{F}-\left(c_{F}-c_{0}\right) \exp \left[-\left(\frac{t}{t_{0}}\right)^{p}\right]$

where $t_{0}$ and $p>1$ are constant coefficients, and $c_{0}$ is the initial silica concentration in the bulk melt. We use $t$ instead of temperature in Eq. (2b) for convenience. For a batch heated at a constant rate, the time term, $t / t_{0}$, can be expressed in terms of temperature $(T)$ as $\left(T-T_{\mathrm{I}}\right) /\left(T_{0}-T_{\mathrm{I}}\right)$, where $T_{\mathrm{I}}$ is the temperature at which $t$ is set at 0 , and $T_{0}$ is the temperature corresponding to $t_{0}$.

The uniform initial mass fraction, $c_{0}$, originated from the batch reactions before the glass-forming melt connected to form a continuous liquid phase. Eq. (2b) describes the situation when, as the temperature increases and dissolution progresses, $c_{\mathrm{B}}$ initially remains at a nearly constant level, $c_{0}$, but eventually begins to increase and approach the level of the final mass fraction, $\mathrm{c}_{\mathrm{F}}$. However, this level is not reached during the particle life because each particle leaves behind an inhomogeneity that only gradually attenuates from the maximum silica mass fraction, $c_{R}$, originally at the quartz-melt boundary.

\subsection{Rate of dissolution and retardation factor}

Because we have disregarded, for simplicity, the difference between the solid and liquid density, we can express the rate of dissolution, $\mathrm{d} R / \mathrm{d} t$, where $R$ is the particle radius, by the equation

$\frac{\mathrm{d} R}{\mathrm{~d} t}=\gamma D \frac{C_{R}-C_{B}}{\delta}$

where $\delta$ is the concentration boundary layer thickness, and $\gamma$ is the dissolution retardation factor that measures the degree of nonideality of the batch. With $\gamma=1$, Eq. (3) becomes the famous NernstNoyes equation, which is based on Fick's law of diffusion.

Eq. (3) can be viewed as a definition of either $\delta$ or $\gamma$. We will assume that initially, when the diffusion-controlled dissolution begins, $\gamma=1$ until increasing $\delta$ reaches the value at which $3 \delta=a / 2-R$. We assume that convection within the bulk melt, caused by the growth and motion of bubbles, maintains either $\delta$, or $R+3 \delta$, depending on the degree of stirring, at a constant level. As soon as $\delta$, or $R+3 \delta$, stops growing, Eq. (3) becomes a definitions of $\gamma$ that begins to decrease to compensate for the rising $D$ that continues to increase with increasing temperature while other variables change little. 
Table 1

Average distances between particles and values of Eq. (2b) coefficients (including auxiliary values).

\begin{tabular}{lclllllll}
\hline $2 R_{0}, \mu \mathrm{m}$ & $a, \mu \mathrm{m}$ & $s_{0}$ & $c_{0}$ & $T_{\mathrm{D}},{ }^{\circ} \mathrm{C}$ & $t_{\mathrm{D}}, \mathrm{h}$ & $p$ & $t_{0}, \mathrm{~h}$ & $T_{0},{ }^{\circ} \mathrm{C}$ \\
\hline 45 & 54 & 0.655 & 0.132 & 1093 & 2.31 & 6.15 & 2.11 & 1033 \\
75 & 90 & 0.768 & 0.093 & 1133 & 2.44 & 5.89 & 2.18 & 1055 \\
150 & 180 & 0.907 & 0.039 & 1266 & 2.89 & 5.07 & 2.48 & 1144 \\
195 & 233 & 0.954 & 0.020 & 1380 & 3.27 & 4.57 & 2.74 & 1222
\end{tabular}

$R_{0}$ is the initial particle size, $a$ is the average distance between centers of neighboring particles.

$s_{0}$ is the undissolved fraction of silica at $700{ }^{\circ} \mathrm{C}, c_{0}$ is the initial silica mass fraction in the bulk melt.

$T_{\mathrm{D}}$ and $t_{\mathrm{D}}$ are the temperature and time at which the particles dissolved.

$p$ and $t_{0}$ are the coefficients in Eq. (2b), and $T_{0}$ is the temperature corresponding to $t_{0}$.

\subsection{Concentration distribution within the concentration layer}

We approximate the concentration distribution around the dissolving particle by an exponential function of the form

$c=c_{B}+\left(c_{R}-c_{B}\right) \exp \left(-\frac{r-R}{\delta}\right)$

where $r$ is the radial distance from the particle center. At this point, by Eq. (4), the value of $c$ at the midpoint between two neighboring particles, i.e., at $r=a / 2$, will increase above $c_{B}$ by $5 \%$ of the $\left(c_{R}-c_{B}\right)$ difference.

\section{Experimental data}

To obtain concrete shapes of the $\gamma(T)$ function, we used experimental data for the dissolution of crushed quartz while heating batches formulated for a high-alumina, high-level waste as reported in $[1,13,14]$. We have selected four batches containing silica particles of $45,75,150$, and $195 \mu \mathrm{m}$. The mass fraction of silica in the glass was $\mathrm{C}_{\mathrm{F}}=0.305$. Assuming that in an ideal batch, the particle size is a diameter of a sphere, then, by Eq. (1), we have $a=2.395 R_{0}$; the $a$ values are listed in Table 1.

Fig. 2 displays the particle sizes, represented by approximation functions defined in [1], versus temperature and time. The time is related to temperature as $t=\left(T-T_{\mathrm{I}}\right) / \Phi$ and is measured from the moment at which the temperature reached the value of $T_{\mathrm{I}}=400{ }^{\circ} \mathrm{C}$. All batches were heated at the constant temperature increase rate of $\Phi=300 \mathrm{~K} / \mathrm{h}$ (starting from room temperature). Note that the rate of dissolution changes little over a wide temperature interval of $\sim 400 \mathrm{~K}$. Fig. 3 presents an example [14] of a measured concentration distribution of silica at the dissolving quartz particle.

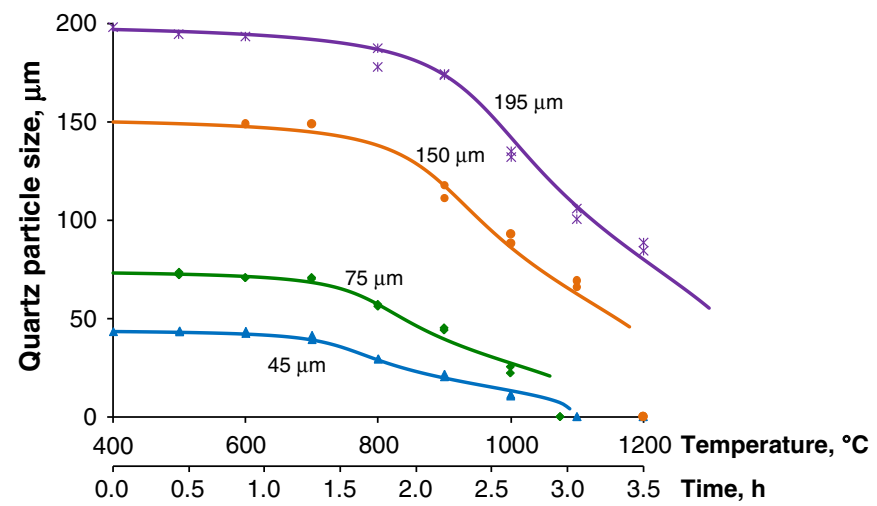

Fig. 2. Effective radius of quartz particles versus time at a constant rate of heating $\left(\mathrm{d} T / \mathrm{d} t=5 \mathrm{~K} / \mathrm{min} ; R^{2}=0.998\right.$, st. error $\left.=0.016 \mu \mathrm{m}\right)$.

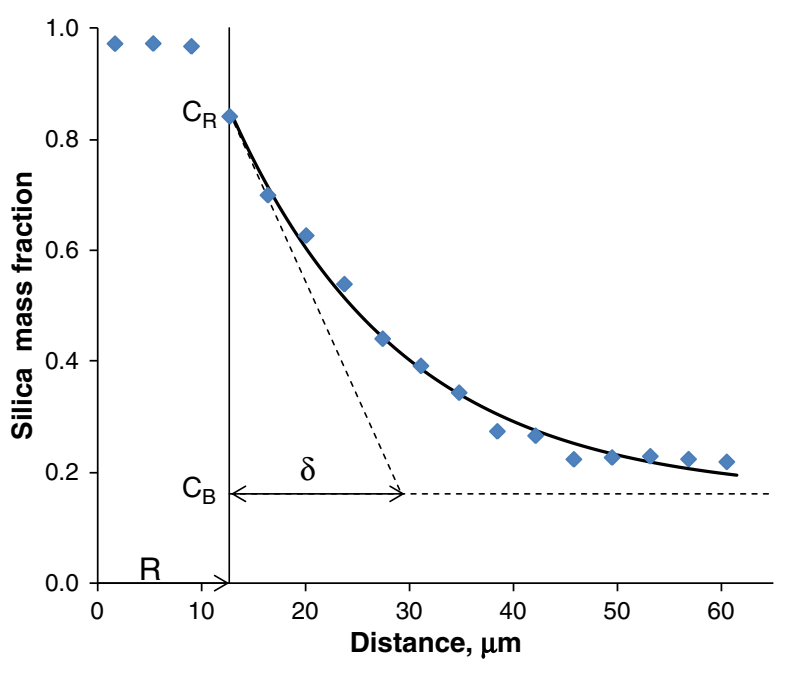

Fig. 3. Concentration distribution of $\mathrm{SiO}_{2}$ at the surface of a dissolving quartz particle; the origin of the $\mathrm{x}$-axis is arbitrary and does not correspond to the particle center $\left(R^{2}=0.992, R_{\mathrm{adj}}^{2}=0.991\right)$.

\section{Results}

To obtain the functions $\delta(T)$ and $\gamma(T)$ by Eq. (3), we need to know or estimate all other variables, i.e., $\mathrm{d} R / \mathrm{d} t, D, c_{\mathrm{R}}$, and $c_{\mathrm{B}}$, as functions of temperature. We obtained the rate of dissolution, $\mathrm{d} R / \mathrm{d} t$, as half of the first derivative of approximation functions defined in [1] and represented by lines in Fig. 2. For the diffusion coefficient,

$D=D_{0} \exp \left(-\frac{B}{T}\right)$

we used the values of $D_{0}=1.122 \times 10^{-5} \mathrm{~m}^{2} \mathrm{~s}^{-1}$ and $B=2.00 \times 10^{4} \mathrm{~K}$. The value of $B$ equals that for the viscosity (as the Stokes-Einstein equation suggests), and $D_{0}$ was estimated based on the concentration profile shown in Fig. 3. Then, as in [1], we assessed $c_{R}$ as

$c_{R}=c_{R 0}+b\left(T-T_{R}\right)$

with $c_{\mathrm{R} 0}=0.610, b=2.67 \times 10^{-5} \mathrm{~K}^{-1}$, and $T_{\mathrm{R}}=273 \mathrm{~K}$.

For $c_{0}$ in Eq. (2b), we used the expression $c_{0}=c_{F}\left(1-s_{0}\right) /\left(1-c_{F} s_{0}\right)$, where $s_{0}$ is the "early" value of the undissolved fraction of total silica after the initial batch reactions were complete, and enough glassforming phase emerged to embed the quartz particles; we used the experimental values of $s_{0}$ for $700{ }^{\circ} \mathrm{C}$ [1].

To select reasonable values for $t_{0}$ and $p$, we imposed two requirements on the $c_{\mathrm{B}}(T)$ function. First, because silica inhomogeneities remain in the melt for some time after all solids dissolve, then $c_{B}$ must be close to but reasonably smaller than $C_{F}$ at the temperature, $T_{\mathrm{D}}$, at which solid silica disappears, or $c_{\mathrm{B}}\left(T_{\mathrm{D}}\right)=\zeta \mathrm{c}_{\mathrm{F}}$, where $\zeta<1$. We used $\zeta=0.9$, i.e., $c_{B}\left(T_{D}\right)=0.275$, for the calculations. Second, we presumed that $c_{\mathrm{B}}$ began to appreciably increase after the temperature reached $700{ }^{\circ} \mathrm{C}$, assuming that $c_{\mathrm{B}}\left(700{ }^{\circ} \mathrm{C}\right)=c_{0}+\left(\mathrm{c}_{\mathrm{F}}-c_{0}\right) \varepsilon$ with $\varepsilon=0.01$, i.e., $C_{\mathrm{B}}\left(700^{\circ} \mathrm{C}\right)$ was above $c_{0}$ by $1 \%$ of the difference between the initial and the final mass fraction of silica in the melt, $c_{F}-c_{0}$. The values of $s_{0}, c_{0}, T_{\mathrm{D}}, t_{\mathrm{D}}$ (the time corresponding to $T_{\mathrm{D}}$ ), $p, t_{0}$, and $T_{0}$, are listed in Table 1.

Fig. 4 displays the $c_{\mathrm{B}}(T)$ functions as estimated for the batches and compares $c_{B}$ estimated by Eqs. (2a) and (2b), showing that $c_{B}$ that is based on mass balance increased faster. Both Eqs. (2a) and (2b), were used for calculating $\delta(T)$ and $\gamma(T)$ functions for vigorous stirring to see the effect of $\mathrm{c}_{\mathrm{B}}(T)$ on the progress of dissolution.

The $\delta(T)$ and $\gamma(T)$ functions, shown in Figs. 5 and 6, were calculated with Eqs. (3), (5), and (6) and the rule that $\delta$ either stops 


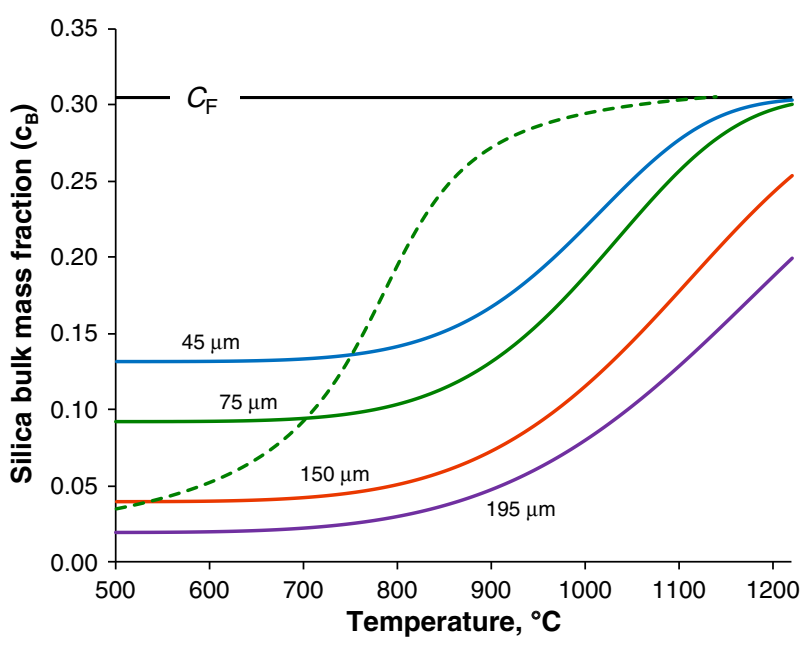

Fig. 4. Silica mass fraction in bulk melt $\left(c_{B}\right)$ versus temperature and initial particle size. The lines represent Eq. (2b) with $p$ and $t_{0}$ coefficients listed in Table 1. The horizontal line indicates the mass fraction of $\mathrm{SiO}_{2}\left(\mathrm{c}_{\mathrm{F}}=0.305\right)$ in the final melt. The dashed line represents Eq. (2a) for $75-\mu \mathrm{m}$ particles.

growing at $3 \delta=a / 2-R$ (vigorous stirring) or continues growing with the rate at which $R$ is receding (mild stirring). The transition from the stage of changing $\delta$ to the stage of changing $\gamma$ (slightly smoothed with a parabolic bridging function) happened between the temperatures $630{ }^{\circ} \mathrm{C}(45 \mu \mathrm{m})$ and $690{ }^{\circ} \mathrm{C}(150$ and $195 \mu \mathrm{m})$. To assess the effect of change of the bulk concentration with temperature, $\delta(T)$ and $\gamma(T)$ were calculated also for the case of vigorous stirring with $c_{\mathrm{B}}(T)$ from Eq. (2a). The $\delta(T)$ thus obtained is virtually indistinguishable from that calculated with $c_{\mathrm{B}}(T)$ defined by Eq. (2b). As shown in Fig. 6b, the difference is significant but small for $\gamma(T)$.

Fig. 7 illustrates the developing concentration profile of silica, by Eq. (4), around a 75- $\mu \mathrm{m}$ quartz particle $\left(R_{0}=37.5 \mu \mathrm{m}\right.$ and $\left.a=90 \mu \mathrm{m}\right)$. The vertical dashed lines indicate the distance of the dissolvingparticle surface from the center corresponding to the measured fractions of dissolving quartz [1]. The horizontal line represents the final silica fraction in the melt $\left(c_{\mathrm{F}}\right)$.

\section{Discussion}

One of the peculiarities of the high-alumina, low-silica glass, the data of which we use in this paper, is that the difference between $c_{R}$

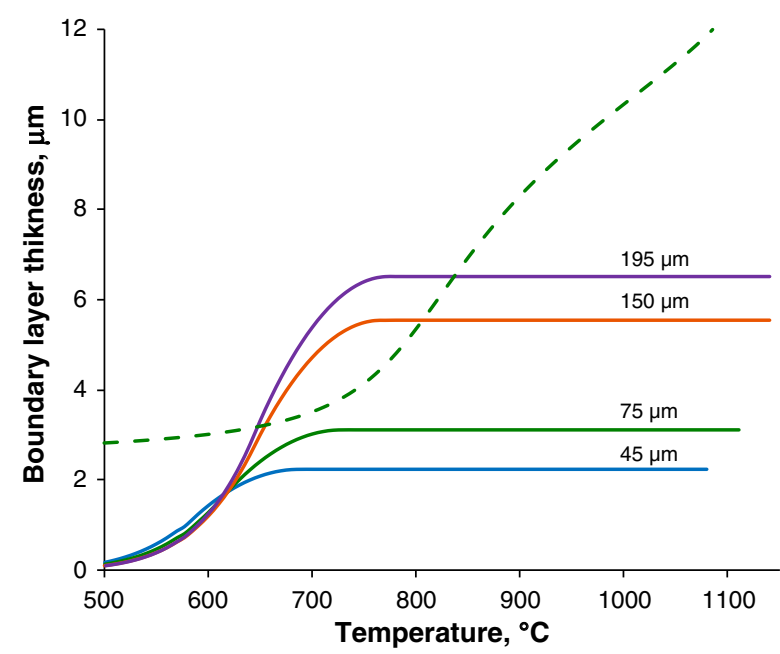

Fig. 5. Concentration layer thickness versus temperature and initial particle size. Solid lines correspond to vigorous stirring and the dashed line to mild stirring for $75-\mu \mathrm{m}$ particles.
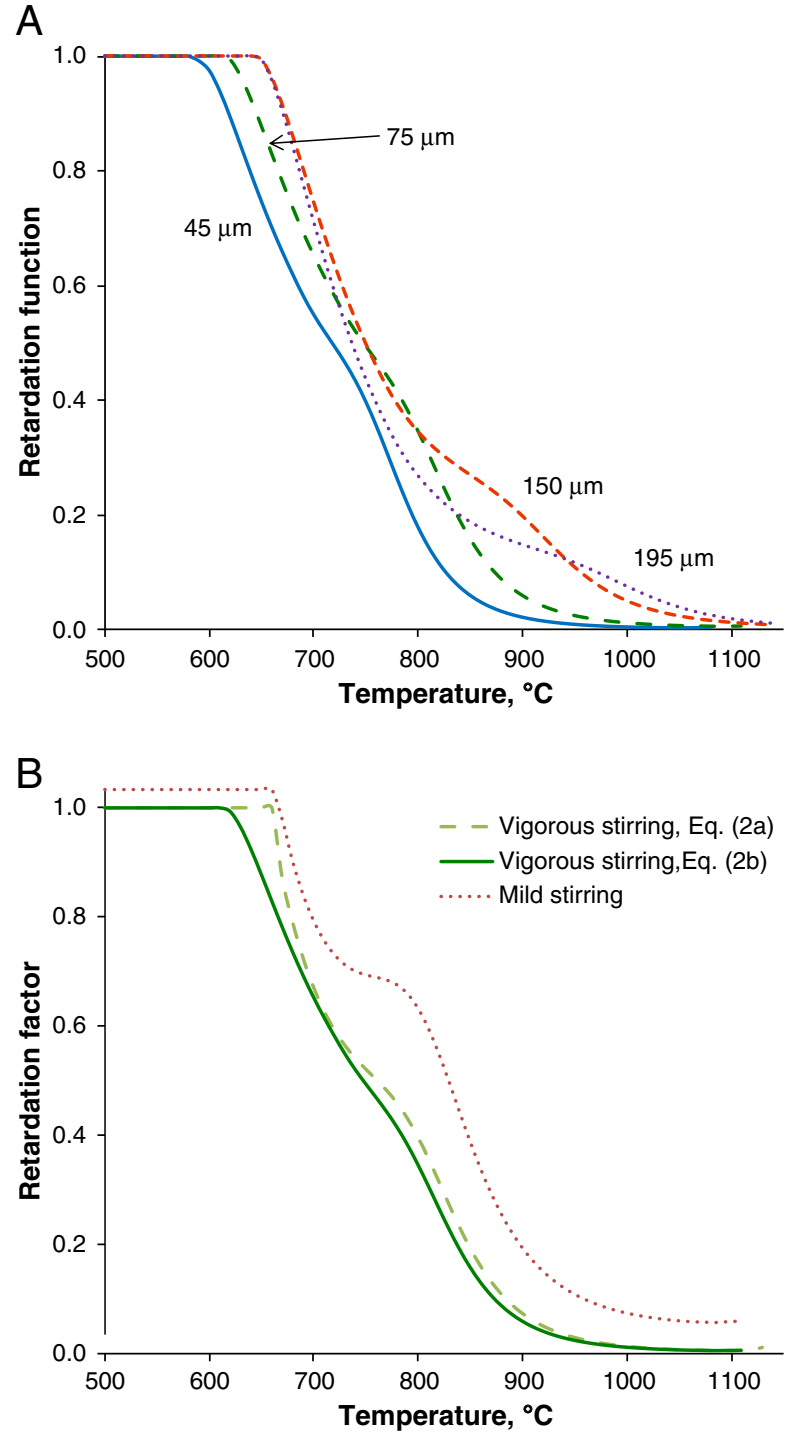

Fig. 6. Retardation factor versus temperature and initial particle size for vigorous stirring (A) and for $75-\mu \mathrm{m}$ particles (B) with mild stirring for $c_{B}$ given by Eq. (2a) (dotted line) and vigorous stirring for $c_{\mathrm{B}}$ given by Eq. (2a) (dashed line) and Eq. (2b) (solid line).

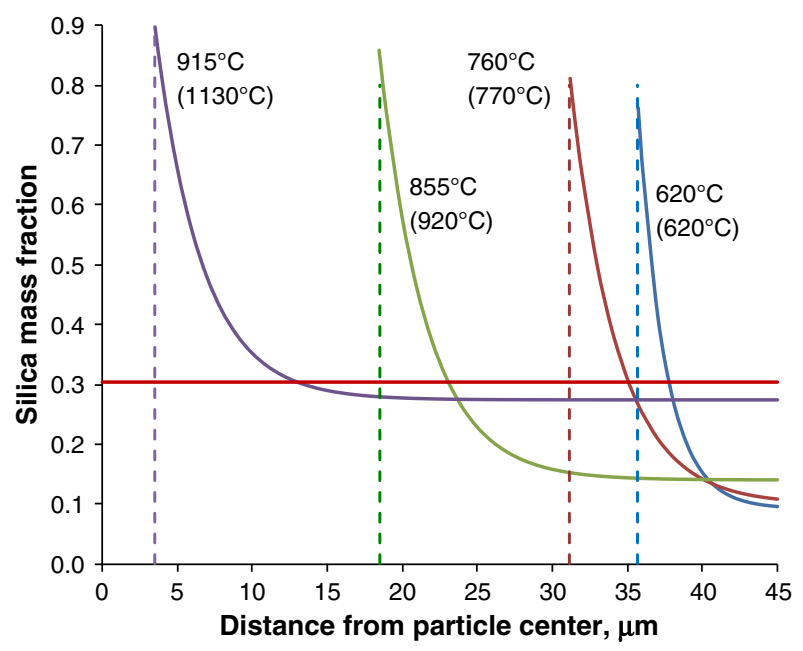

Fig. 7. Silica concentration distribution around a 75- $\mu \mathrm{m}$ dissolving particle in an ideal batch during vigorous stirring at temperatures as indicated; the real batch temperatures corresponding to the same degree of dissolution are in parentheses. The horizontal line shows the $\mathrm{SiO}_{2}$ mass fraction in the final glass $\left(\mathrm{c}_{\mathrm{F}}\right)$. 
and $\mathrm{C}_{\mathrm{F}}$ is relatively large. As a result, by Eq. (2a), the difference between $\mathrm{SiO}_{2}$ mass fraction at the particle surface and the average mass fraction of silica in the bulk melt, i.e., $c_{R}-c_{B}$, is always large because the average $c_{\mathrm{B}}$ never exceeds $c_{\mathrm{F}}$ during the particles' life. Consequently, the $\mathrm{SiO}_{2}$ concentration in the average bulk cannot become close to saturation, and thus cannot be responsible for retarding the dissolution. Because the thickness of the concentration layers is limited by the distance between particles, $\delta$ cannot become large enough to slow dissolution through a low concentration gradient at the interface; $\mathrm{dc} /\left.\mathrm{d} r\right|_{\mathrm{r}=\mathrm{R}}=\left(c_{\mathrm{R}}-c_{\mathrm{B}}\right) / \delta$. Though the value of $D$ would somewhat increase with increasing $c_{\mathrm{R}}$, the effect is small compared to the increase of $D$ with increasing temperature. Although, by definition, there is no difference between the real batch and the ideal batch initially (for 75- $\mu$ m particles, $3 \delta$ reaches the thickness of $a / 2-R$ at $660{ }^{\circ} \mathrm{C}$; thus $\gamma$ becomes $<1$ above $660{ }^{\circ} \mathrm{C}$ ), in the advanced stages, the quartz particles dissolve much slower in the real batch than in the ideal-batch. With $\gamma=1$, the $75-\mu \mathrm{m}$ particles would fully dissolve by $920^{\circ} \mathrm{C}$ in the ideal-batch instead of at the measured $T_{\mathrm{D}}$ of $1133^{\circ} \mathrm{C}$ [1], see Table 1 . The temperatures at the profiles in Fig. 7 indicate the dissolution progress of both the real (in parentheses) and the ideal batch.

In the ideal batch, the particles are regularly distributed in space and dissolve in a symmetric manner, i.e., the thickness of concentration layers around the particles is independent of the position on the particle surface. During the initial phase of dissolution, where $\gamma=1$, the real batch is ideal in the sense that the concentration layers are thin as compared to the distances between the particles and thus increase around each particle independently of the presence of other particles in the melt. At this stage, the geometric arrangement of particles is irrelevant as soon as the particles are separated by the melt. In the real batch, the departure from the ideal behavior begins when the dissolving particles influence each other through their concentration layers, i.e., when the concentration layer around a particle impinges on those of neighboring particles. Afterward, as in the ideal batch, the $c_{\mathrm{B}}$ grows, but not in a symmetrical manner. In an ideal batch, we assume that convection keeps the bulk melt at a uniform concentration and prevents concentration layers from extending beyond a certain distance. However, in the real batch, the silica concentration in the melt can grow to a level close to saturation, instead of keeping below $c_{F}$, at areas where the particle surfaces are close to each other. In such areas, high concentrations of silica persist because convection ceases in them as a result of a high viscosity and a lack of moving bubbles. Hence, dissolution virtually stops in these areas. The stirring action of bubbles is limited to the low-viscosity channels through which they travel to the melt surface.

Accordingly, inhibited dissolution results from particle interactions. As the particles are more and more pushed to clusters by moving bubbles, the $\gamma$ factor, the measure of the extent of this inhibition, decreases from 1 (the uninhibited dissolution) to values close to 0.01 (see Fig. 6) while the rate of dissolution, measured as $\mathrm{d} R$ / $\mathrm{dt}$, changes little over the span of $400 \mathrm{~K}$, even though $D$ increases by two orders of magnitude.

One can consider additional causes of the impeded dissolution:

1) The surface-to-volume ratio of crushed quartz particles, whose shapes are highly irregular, is large. Even though the particle surfaces are smoothed by the early reactions, and the particles become more rounded before the melt becomes continuous, particle surfaces continue to decrease, not only because of the decrease in volume, but also because the surface curvature becomes more and more uniform. However, this process could hardly cause the severe decrease of $\gamma$ seen in Fig. 6 .

2) The width of the particle-size distribution results in an early increase of the bulk concentration and thus a decrease of the driving force of dissolution, $c_{R}-c_{B}$. Although the smallest particles, debris, are removed by the early reactions, the particle-size distribution would result in a decreased number of particles toward the end of the process. However, the decreasing driving force can contribute little to the massive dissolution retardation observed. Though larger particles can exist longer, they cannot persist long enough to cause the two orders of magnitude decrease in $\gamma$.

Consequently, the impact of the nonuniform spatial distribution of particles on the concentration field within the melt is mainly responsible for the large extent of dissolution retardation. Dissolution slows down or even stops at a portion of particle surfaces in the nearly saturated melt between close particles. Moreover, particles inside aggregates may not dissolve at all until the inhomogeneities around them are dispersed. Inhomogeneities with a high concentration of silica possess a high viscosity, and they resist stretching by convection. Therefore, particles embedded in them survive until the temperature is sufficiently high, and the inhomogeneities attenuate by convective diffusion.

The open question remains as to how the extended lifetime of particles, caused by the dissolution-inhibiting factors, impacts the rate of glass processing in continuous melters. Because of retarded dissolution, a higher temperature and a longer time are needed to dissolve the last surviving particles and disperse the inhomogeneities. However, slow dissolution does not necessarily result in a slower processing rate. Some effects of the presence of solid inclusions at a high temperature are clearly beneficial. Delayed dissolution allows the bulk of the melt to maintain a low viscosity, resulting in a faster removal of bubbles and a lower tendency to create foam [13].

As a final note, one of the reviewers of this paper pointed out that the melt basicity decreases as the $\mathrm{SiO}_{2}$ concentration rises, causing the solubilities of gasses, such as $\mathrm{CO}_{2}$ and $\mathrm{SO}_{2}$, to decrease. Tiny bubbles may form on the last residues of solid particles and be trapped in the viscous inhomogeneities. Hence, the retarded dissolution of quartz particles, though allowing an easy escape of larger bubbles from the bulk melt, can obstruct fining by forming hard-to-remove bubbles in silica-rich regions.

\section{Conclusions}

Compared to an ideal batch with monodisperse spherical particles that are uniformly distributed and uniformly dissolve in a melt homogenized by the stirring effect of gas bubbles, the observed dissolution of quartz particles in a real batch appears severely inhibited. The dissolution retardation factor defined by Eq. (3) decreased by two orders of magnitude from the initial value of 1 to the final value close to 0.01 . This inhibition can be attributed mainly to the irregular spatial distribution of the particles, although the width of the particle-size distribution and irregular shapes of the particles may also play a role. The delayed dissolution allows the bulk of the melt to maintain a low viscosity, resulting in a faster removal of bubbles and a lower tendency to create foam, but the presence of high-silica inhomogeneities unfavorably affects the solubility of gasses.

\section{List of symbols}

a the average distance between centers of neighboring particles, $\mathrm{m}$ activation energy in Eq. (5), K coefficient in Eq. (6), $\mathrm{K}^{-1}$ initial silica mass fraction in the bulk melt silica bulk mass fraction silica final mass fraction after all particles dissolve and inhomogeneities disappear

saturated (equilibrium) silica mass fraction at the quartzmelt boundary coefficient in Eq. (6)

${ }_{S} \quad$ undissolved silica fraction in the mixture of quartz particles and melt 
Note: Whenever convenient, the temperature was measured in ${ }^{\circ} \mathrm{C}$, time in hours, and distances in $\mu \mathrm{m}$ or $\mathrm{mm}$.

\section{Acknowledgments}

The authors are grateful to the U.S. Department of Energy's Hanford Tank Waste Treatment and Immobilization Plant Federal
Project Office, Engineering Division, for financial support and Albert Kruger for his assistance and guidance. The authors would like to thank Dong-Sang Kim and Mike Schweiger for inspiring discussions. Pacific Northwest National Laboratory is operated for the U.S. Department of Energy by Battelle under Contract DE-AC05-76RL01830. In its final stage, this work was supported by a World Class University program through the National Research Foundation of Korea funded by the Ministry of Education, Science and Technology (R31-30005).

\section{References}

[1] P. Hrma, K.J. Swearingen, S.H. Henager, M.J. Schweiger, J. Marcial, N.E. TeGrotenhuis, J. NonCryst. Solids (2010) (accepted).

[2] D.W. Ready, A.R. Cooper, Chem. Engng. Sci. 21 (1966) 917.

[3] K.G. Kreider, A.R. Cooper, Glass Technol. 8 (1967) 71

[4] M. Cable, D.J. Evans, J. Appl. Phys. 38 (1967) 2899.

5] J.L. Duda, J.S. Vrentas, Int. J. Heat Mass Transfer 14 (1971) 395.

[6] Physicochemical Hydrodynamics, Prentice-Hall, Englewood Cliffs, New York, 1962, p. 80.

[7] A.W. Hixson, J.H. Crowell, Ind. Eng. Chem. 23 (1931) 923

[8] J. Alton, T.J. Plaisted, P. Hrma, Chem. Engn. Sci. 57 (2002) 2503.

[9] P. Hrma, Silikáty 24 (1980) 7.

[10] L. Němec, J. Amer. Ceram. Soc 60 (1977) 436

[11] J. Kloužek, M. Arkosinová, L. Němec, P. Cincibusová, Eur. J. Glass Sci. Technol. A 48 (2007) 176.

[12] L. Němec, P. Cincibusová, Ceramics Silikáty 53 (2009) 145.

[13] S.H. Henager, P. Hrma, K.J. Swearingen, M.J. Schweiger, J. Marcial, N.E. TeGrotenhuis, J. NonCryst. Solids 357 (2011) 829.

[14] M.J. Schweiger, P. Hrma, C.J. Humrickhouse, J. Marcial, B.J. Riley, N.E. TeGrotenhuis, J. NonCryst. Solids 356 (2010) 1359. 